\title{
Evolution récente et future des paysages sylvo-pastoraux du Jura vaudois
}

\author{
Alexandre Buttler \\ Thomas Spiegelberger \\ Joël Chételat \\ Michael Kalbermatten \\ Katy Lannas \\ Alexander Peringer \\ Jean-Bruno Wettstein \\ François Gillet
}

\author{
Laboratoire des systèmes écologiques, EPFL et Institut fédéral de recherches WSL $(\mathrm{CH})^{*}$ \\ Research Unit Mountain Ecosystems, Irstea et EPFL (CH) \\ Microgis Foundation for Spatial Analysis MFSA (CH) \\ Laboratoire des systèmes écologiques, EPFL $(\mathrm{CH})$ \\ Laboratoire des systèmes écologiques, EPFL $(\mathrm{CH})$ \\ Institute for Landscape Planning, University of Stuttgart ILPOE (D) et EPFL (CH) \\ Bureau d'agronomie, Ste-Croix $(\mathrm{CH})$ \\ Université de Franche-Comté, Besançon (F) et EPFL (CH)
}

\begin{abstract}
Recent and future evolution of the silvopastoral landscape in the Jura vaudois
Land-use management in silvopastoral landscapes is particularly complex because such ecosystems depend on a subtle equilibrium in the management scheme which aims to provide various ecological goods and services such as fodder, timber and biodiversity, as well as areas for leisure and attractive scenery. In this study we investigated land-use changes in the Parc naturel régional Jura vaudois, and the changes in tree cover density in two pastures using land-use statistics and aerial photographs. A prospective modelling approach was also undertaken on one pasture to assess changes in the landscape structure under two climate scenarios. A general tendency towards segregation between closed forests and open pastures was observed. The agriculture policy has markedly influenced the dynamics of tree cover density, but this effect depended very much on the local situation, for example, the proximity of a village, which allows a better optimization of farm management. The simulation of future development of these landscapes suggests that one might expect large changes in the structure and composition of the vegetation because of climate change. In the long run, areas of high tree densities will expand, and spruce will give way to beech and pine, depending on the warming intensity. Nevertheless, adaptation to new environmental conditions for sustaining ecological goods and services will call for management measures appropriate to the intensity of climate change. Furthermore, an active adaptive management based on experimentation and innovation, as well as allowing collaboration between scientists and land managers will be required to face and mitigate the ecological problems associated with climate change.
\end{abstract}

Keywords: wooded pastures, silvopastoral system, forest dynamics, climate change doi: $10.3188 /$ szf.2012.0469

*ENAC, ECOS, Station 2, CH-1015 Lausanne, courriel alexandre.buttler@epfl.ch
$\mathrm{L}$ es écosystèmes sylvo-pastoraux tels que les pâturages boisés forment des paysages traditionnels en Europe (Etienne 1996), mais nombreux sont ceux qui ont disparu au XXe siècle (Gillet \& Gallandat 1996). Gallandat et al (1995) ont montré que les pâturages boisés des montagnes suisses sont menacés par une évolution divergente: les arbres envahissent les zones insuffisamment productives ou trop éloignées des fermes, que les agriculteurs ont tendance à abandonner, alors que dans les zones plus favorables, qui permettent une exploitation plus intensive, les arbres et arbustes sont de moins en moins nombreux, et leur régénération est insuffisante. Une des conséquences en est l'appauvrissement de la biodiversité et une perte d'attractivité du paysage.
Dans le Jura suisse, les pâturages boisés se trouvent à l'étage montagnard et subalpin, essentiellement entre $800 \mathrm{~m}$ et $1400 \mathrm{~m}$ d'altitude (Gillet \& Gallandat 1996). Le climat y est relativement froid; par exemple, à $1200 \mathrm{~m}$, la température moyenne journalière est inférieure à $0{ }^{\circ} \mathrm{C}$ pendant plus de 60 jours par année, et l'enneigement peut durer de décembre à avril. Le climat de ces régions montagnardes limite le pastoralisme à la période estivale, généralement de mai à septembre. Le bétail est le plus souvent composé de génisses, de vaches laitières ou allaitantes, et de chevaux dans certaines régions.

Quatre types structuraux principaux forment la végétation (Gallandat et al 1995, Vittoz 1998): les pâturages non boisés (catégorie 1000), avec moins 
de $1 \%$ de recouvrement d'arbres, les pâturages faiblement boisés (catégorie 2000), avec 1 à 20\% de couverture d'arbres qui sont pour la plupart isolés, les pâturages densément boisés (catégorie 3000), avec 20-70\% de couverture d'arbres qui sont le plus souvent agrégés en bosquets, et les forêts pâturées (catégorie 4000), avec plus de 70\% de couverture arborescente.

Un inventaire (Gallandat et al 1995) a montré qu'un sixième de la flore vasculaire de Suisse se retrouve dans les pâturages boisés du Jura suisse. La biodiversité végétale est également très riche sur des surfaces restreintes, jusqu'à 40 espèces de plantes par $\mathrm{m}^{2}$ (Buttler et al 2009). L'origine de cette grande richesse d'espèces végétales est multiple et peut s'expliquer par des processus à différentes échelles. Elle tient en premier lieu à l'importante variabilité spatiale des sols allant des sols calcaires superficiels aux sols profonds, acides et limoneux (Havlicek et al 1998, Gobat et al 1989). Deuxièmement, une mosaïque complexe d'arbres, d'arbustes et d'herbages ouverts crée des microclimats variés qui favorisent le développement de différentes espèces végétales. Il a par exemple été démontré que la richesse spécifique de la strate herbacée est maximale avec une couverture de 30\% d'arbres (Gillet et al 1999). Troisièmement, l'activité du bétail peut avoir un impact sur la composition floristique (Kohler et al 2004a, 2004b, 2006a, 2006b, Smit et al 2005, Gillet et al 2010), sur la régénération des arbres (Vandenberghe et al 2006, 2007a, 2007b, 2009) ainsi que sur le sol (Kohler et al 2005). Enfin, la richesse spécifique végétale est liée à la complexité topographique, selon les variations du microrelief et la configuration spatiale (Dufour et al 2006). Les pâturages boisés sont donc un bon exemple de paysage où une biodiversité importante peut coexister avec une utilisation agricole extensive.

L'utilisation de tels écosystèmes semi-naturels est particulièrement délicate, car leur existence dépend du subtil équilibre de leur gestion qui vise à fournir différents biens et services écologiques, allant de la production d'herbe et de bois à la promotion de la biodiversité, ainsi qu'à la mise à disposition d'aires de détente et de paysages attractifs dont l'importance sociale a été démontrée (Miéville-Ott $\&$ Barbezat 2005). Cet aspect revêt une importance croissante en raison des mutations en cours dans les pratiques de l'agriculture de montagne face aux contraintes socio-économiques et aux changements climatiques. La gestion forestière vise actuellement essentiellement à maintenir la couverture forestière avec sa composante hétérogène. Le forestier intervient dans les pâturages peu boisés pour éliminer les arbres dépérissants et pour assurer une régénération suffisante. La rentabilité de l'exploitation du bois, mise à mal dans les temps récents, n'est pas la première priorité dans les pâturages peu boisés, mais peut l'être dans les zones davantage boisées.
Compte tenu de la valeur socio-économique, patrimoniale, écologique, touristique et paysagère des pâturages boisés du Jura, une gestion intégrée est requise, qui doit faire collaborer les forestiers, les agronomes, les écologues et les socio-économistes pour une gestion optimisée (Gmür \& Wettstein 1986, Gmür et al 1989, Perrenoud et al 2003). A cet égard, des outils de gestion ont été développés (Barbezat \& Boquet 2008) pour inciter les gestionnaires à passer d'un mode de gestion sectoriel à un mode intégré, tenant compte de la multifonctionalité de ces milieux et de la nécessité de respecter les différents intérêts en jeu, en maintenant à la fois les perspectives conservationnistes (biodiversité, paysages) et utilitaires (production de biens économiques). Ces réflexions devront également et de plus en plus considérer les changements climatiques qui nécessiteront une gestion adaptative. Le projet «Mountland», du Centre de compétence pour l'environnement et la durabilité du domaine des Ecoles polytechniques fédérales, avait précisément pour but de développer des pratiques innovantes et durables d'utilisation du territoire en région de montagne, dans un contexte de changements globaux et de réchauffement climatique. Les questions qui se posent pour les pâturages boisés du Jura sont: Comment le paysage a-t'il évolué au cours des dernières décennies? Que deviendront ces paysages sous l'effet des changements climatiques? Quelles seront les espèces d'arbres qui seront favorisées? Quelles mesures de gestion forestière pourraient permettre de limiter les impacts sur les écosystèmes et le paysage?

\section{Analyse rétrospective du paysage}

Au cours des dernières décennies, la superficie couverte par les pâturages boisés (ou prés-bois) en Europe a diminué de façon drastique en raison de changements dans les pratiques d'utilisation des terres, en particulier liés à l'intensification agricole locale et à l'abandon (Etienne 1996, Gallandat et al 1995). Les changements d'occupation du sol et d'évolution des surfaces occupées par les pâturages boisés ont été analysés au moyen de deux approches différentes dans le cadre du projet «Mountland». A l'échelle du territoire du Parc naturel régional Jura vaudois (PJV), les statistiques suisses de la superficie (OFS 2001) ont été utilisées pour évaluer les changements intervenus entre 1985 et 2009 sur 11 catégories d'utilisation du sol. A une échelle plus fine, celle de deux pâturages, Les Planets près du village de Bullet (altitude moyenne: $1225 \mathrm{~m}$, figure 1), et Rionde Dessus dans la Combe des Amburnex près du col du Marchairuz (altitude moyenne: $1307 \mathrm{~m}$, figure 2), une deuxième étude a permis de mettre en évidence, au moyen d'une analyse comparative de photographies aériennes des années 1934, 1954, 1968, 1985 


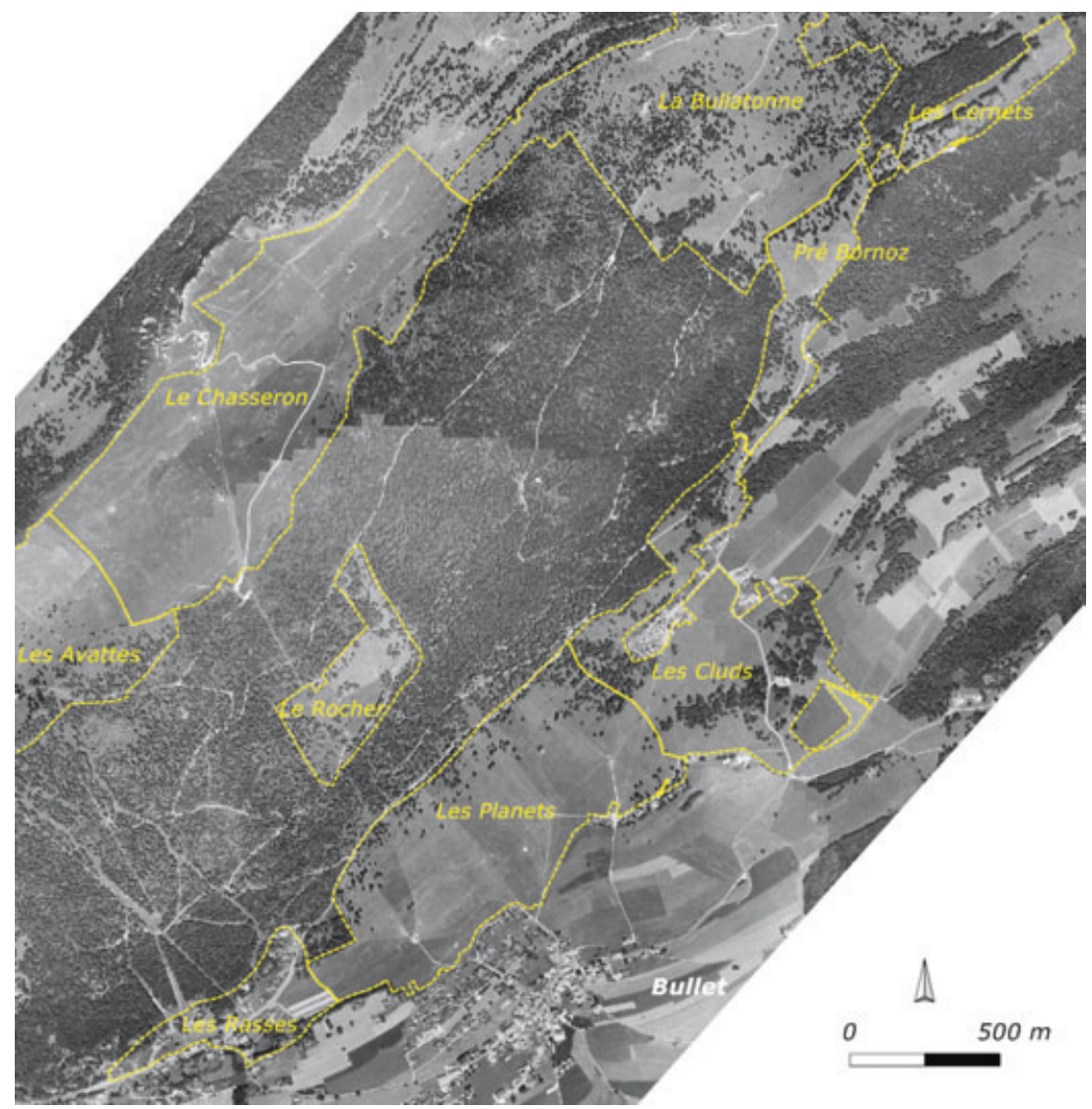

Fig. 1 Site de Bullet comprenant les unités d'exploitation Les Rasses, Les Planets et Les Cluds en I'an 2000. Photo: Swisstopo, autorisation 5701104246 / 000010.

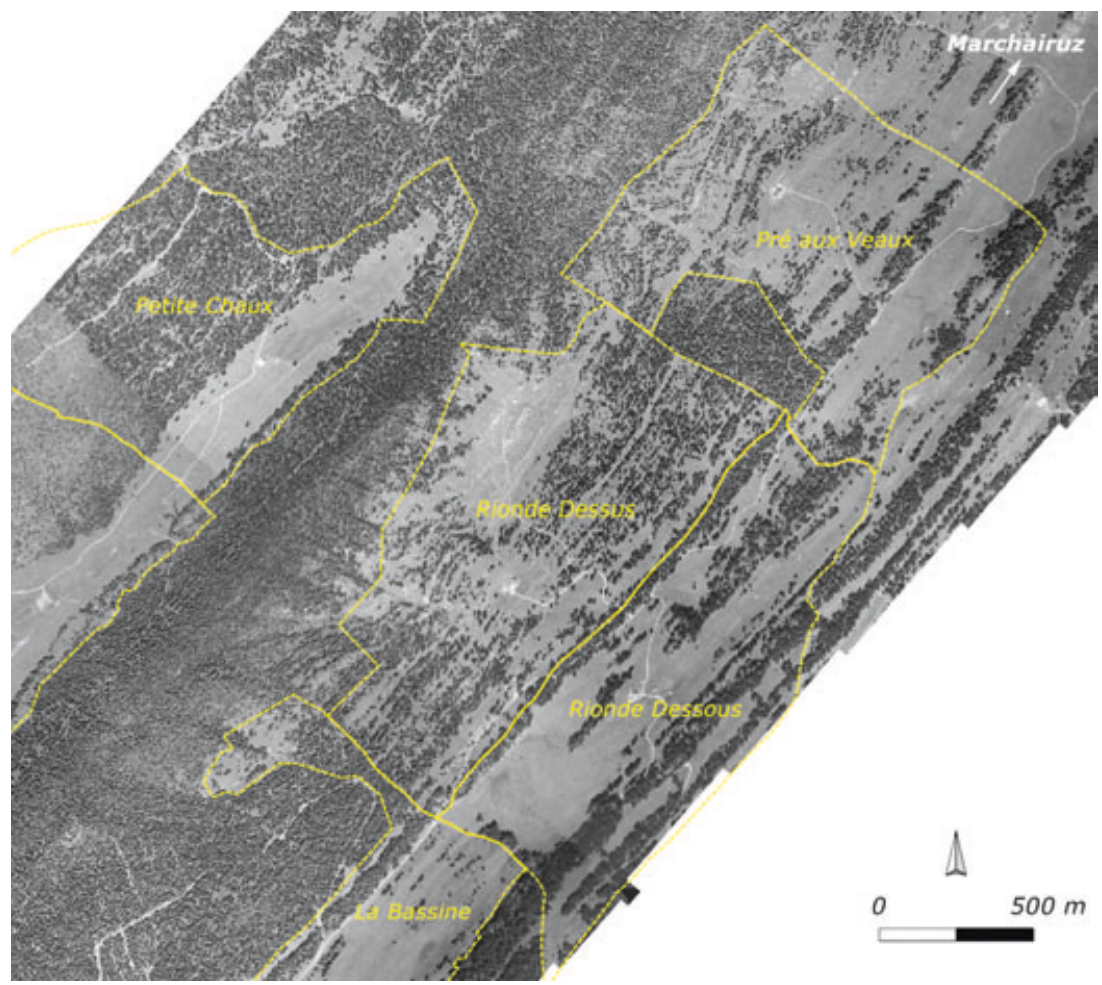

Fig. 2 Site des Amburnex (Marchairuz) comprenant les unités d'exploitation Rionde Dessus, Rionde Dessous et Pré aux Veaux en I'an 2000. Photo: Swisstopo, autorisation 5701104246 / 000010.
Statistiques d'utilisation du territoire du Parc naturel régional Jura vaudois

Le territoire du PJV constitue une région représentative du paysage du Jura suisse qui sera labellisée en début 2013 «Parc naturel régional» et qui comprend 31 communes sur un territoire totalisant $542 \mathrm{~km}^{2}$. Pour quantifier les changements intervenus dans les 25 dernières années dans le PJV, trois enquêtes statistiques de la superficie ont été utilisées pour calculer le nombre, la direction et le type de changements parmi différentes catégories d'utilisation du sol entre les périodes 1985-1997 et 19972009. Les statistiques se font à partir de photographies aériennes en utilisant une grille de points d'échantillonnage distants de $100 \mathrm{~m}$, donnant ainsi un type d'utilisation du sol pour chaque hectare. La couverture du sol à chaque point est déterminée en fonction d'une typologie comprenant 72 classes (OFS 2001). Dans cette étude, ces catégories ont été agrégées en 11 classes pour mettre l'accent sur le gradient entre forêt et pâturage (tableau 1). Dans l'analyse des matrices de transition d'une période à l'autre, tout changement menant vers davantage de couverture forestière est qualifié d'extensification d'utilisation du sol, tandis que la tendance inverse est qualifiée d'intensification.

La tendance générale de 1985 à 2009 (tableau 2) est une augmentation, dans les proportions relatives, de la forêt dense (+2.8\% de 1985 à 2009) et des zones urbaines $(+0.7 \%)$ avec des pertes concomitantes de «coupes et surfaces forestières dévastées» $(-1.0 \%)$, forêts clairsemées $(-0.7 \%)$, terres arables $(-0.6 \%)$, pâturages $(-0.5 \%)$, prairies $(-0.3 \%)$ et pâturages boisés $(-0.2 \%)$.

Pour une analyse plus fine, l'échantillon a été subdivisé en deux zones géographiques: une région de montagne et une région de basse altitude. La région de montagne (altitude supérieure à $1000 \mathrm{~m}$ ) se compose principalement de forêts et de pâturages, et inclut la crête du Jura avec ses hauts plateaux et la Vallée de Joux; elle est caractérisée par la rareté des terres arables et des zones urbaines. La région de basse altitude inclut le flanc sud-est du PJV formant les contreforts du Jura bordant le Plateau suisse. Cette région étant sous l'influence du voisinage des grandes villes, son utilisation des terres est davantage marquée par les zones bâties et les terres arables.

Les régions se distinguent par des différences dans les processus d'extensification et d'intensification. La région de plaine (figure 3) montre un taux important de transformations, notamment dans la première période de 1985 à 1997. Les transformations vers les zones urbaines totalisent $+10.9 \%$, dont $5.4 \%$ par transformation des terres agricoles en zone urbaine. Cette tendance s'est légèrement affaiblie dans la seconde période 1997-2009 (+7.1\%): les plus grands changements vers une extensification ont concerné la transformation de terres agricoles et de 


\begin{tabular}{|c|c|c|}
\hline Numéro & Classe après agrégation & Catégories de base de la Statistique suisse de la superficie (OFS, NOAS04) \\
\hline 1 & Zones urbanisées & (27 catégories non présentées ici) \\
\hline 2 & Terres agricoles & 41 Terres arables \\
\hline \multirow[t]{2}{*}{3} & \multirow[t]{2}{*}{ Prairies } & 42 Prairies naturelles \\
\hline & & 45 Alpages fauchés \\
\hline \multirow[t]{3}{*}{4} & \multirow[t]{3}{*}{ Pâturages } & 43 Pâturages locaux \\
\hline & & 44 Prés et pâturages locaux embroussaillés \\
\hline & & 46 Alpages favorables \\
\hline \multirow[t]{3}{*}{5} & \multirow[t]{3}{*}{ Pâturages embroussaillés } & 47 Alpages embroussaillés \\
\hline & & 48 Alpages rocailleux \\
\hline & & 49 Alpes à moutons \\
\hline \multirow[t]{2}{*}{6} & \multirow[t]{2}{*}{ Groupes d'arbres, pâturages boisés } & 59 Groupes d'arbres (sur surfaces agricoles) \\
\hline & & 60 Groupes d'arbres (sur surfaces improductives) \\
\hline \multirow[t]{2}{*}{7} & \multirow[t]{2}{*}{ Forêt clairsemée } & 55 Forêt clairsemée (sur surfaces agricoles) \\
\hline & & 56 Forêt clairsemée (sur surfaces improductives) \\
\hline \multirow[t]{2}{*}{8} & \multirow[t]{2}{*}{ Forêt dense } & 50 Forêt normale \\
\hline & & 51 Forêt étroite \\
\hline 9 & Aires afforestées & 52 Aires afforestées \\
\hline \multirow[t]{2}{*}{10} & \multirow[t]{2}{*}{ Coupes et surfaces forestières dévastées } & 53 Coupes de bois \\
\hline & & 54 Surfaces forestières dévastées \\
\hline 11 & Autres catégories & (26 catégories non présentées ici) \\
\hline
\end{tabular}

Tab. 1 Agrégation des classes d'utilisation des sols de la typologie de l'Office fédéral des statistiques (OFS, NOASO4).

\begin{tabular}{|c|c|c|c|c|}
\hline Numéro & Classe & $\begin{array}{c}\text { Proportion } 1985 \\
(\%)\end{array}$ & $\begin{array}{c}\text { Proportion } 1997 \\
(\%)\end{array}$ & $\begin{array}{c}\text { Proportion } 2009 \\
(\%)\end{array}$ \\
\hline 1 & Zones urbanisées & 2.2 & 2.7 & 2.9 \\
\hline 2 & Terres agricoles & 12.2 & 11.9 & 11.6 \\
\hline 3 & Prairies & 5 & 4.9 & 4.7 \\
\hline 4 & Pâturages & 18.2 & 17.8 & 17.7 \\
\hline 5 & Pâturages embroussaillés & 0.3 & 0.3 & 0.3 \\
\hline 6 & Groupes d'arbres, pâturages boisés & 2.5 & 2.4 & 2.3 \\
\hline 7 & Forêt clairsemée & 5.4 & 4.8 & 4.7 \\
\hline 8 & Forêt dense & 44.8 & 47.7 & 47.6 \\
\hline 9 & Aires afforestées & 0.2 & 0.2 & 0.1 \\
\hline 10 & Coupes et surfaces forestières dévastées & 3.1 & 1.3 & 2.1 \\
\hline 11 & Autres catégories & 6.1 & 6 & 6 \\
\hline
\end{tabular}

Tab. 2 Proportions relatives des différentes catégories d'utilisation des sols dans le Parc naturel régional Jura vaudois, calculées selon le nombre de points d'échantillonnage dans chaque catégorie.

prairies en pâturages $(+8.5 \%)$, ainsi que la forte dégradation de la forêt dense en classe «coupes et forêts dévastées» (+36.5\%), probablement principalement causée par la tempête Lothar qui a eu lieu en décembre 1999.

La région de montagne a montré une dynamique différente par rapport à la région de basse altitude (figure 4). Seuls $4.5 \%$ des transformations se font en direction des zones urbanisées au cours des deux périodes considérées, contre $18.0 \%$ dans la zone de plaine, ce qui montre une tendance vers une urbanisation accrue en zone de plaine. En région de montagne, le processus d'extensification prévaut: durant la période 1985-1997, les sites d'estivage comme les pâturages se sont transformés en pâturages boisés (7.3\%) qui eux ont également subi une évolution vers les forêts clairsemées (7.3\%). Mais le plus grand changement (25.2\%) concerne la transformation des forêts clairsemées en forêts denses. Pour la deuxième période, les tendances sont semblables, mais moins prononcées.

\section{Analyse des photographies aériennes des pâturages}

L'objectif de la deuxième étude ayant été d'examiner, à une échelle plus fine, l'évolution de la forêt et des pâturages en relation avec les phénomènes naturels (sécheresses, ouragans), les effets des politiques environnementales et du contexte socioéconomique, moteur important des pratiques de gestion, nous avons croisé l'interprétation des photographies aériennes avec les connaissances du 


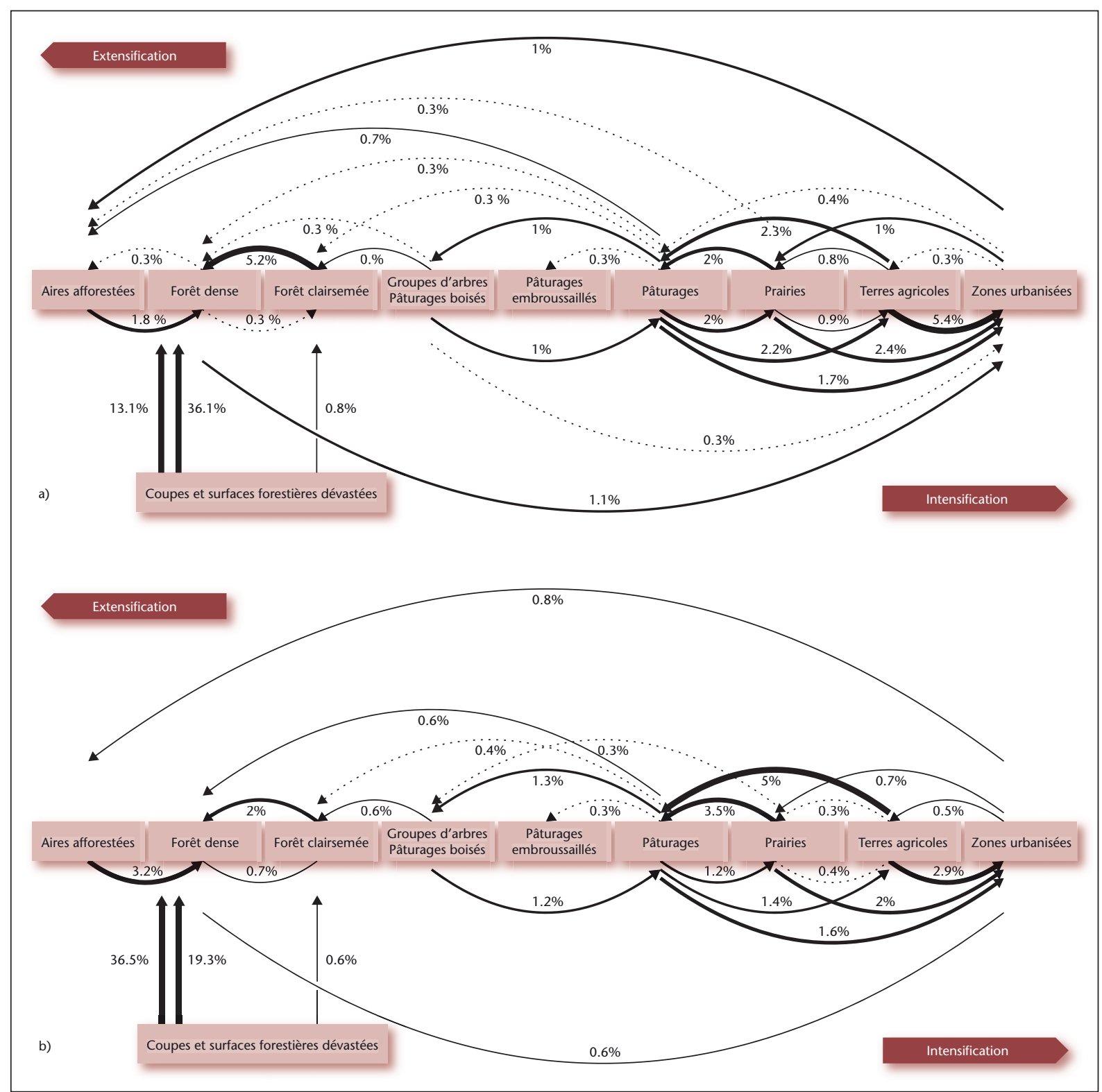

Fig. 3 Changement d'utilisation des sols dans les zones de basse altitude du Parc naturel régional Jura vaudois (à une altitude inférieure à 1000 m). A: période 1985 à 1997, B: période 1997 à 2009. Les valeurs représentent les taux de changement en \% par rapport à l'ensemble des changements durant la période considérée (A: 2381 points, B: 2064 points). Les taux inférieurs à 0.2\% ne sont pas indiqués.

contexte historique. En plus des jalons liés à l'évolution générale de la politique foncière suisse, nous avons compilé les chroniques sur la gestion des pâturages des deux sites d'étude sur la base des archives (plans de gestion) et des entrevues avec des représentants du monde agricole et sylvicole (propriétaires, forestiers, agriculteurs). La densité de la couverture forestière dans les deux unités d'exploitation (Les Planets et Rionde Dessus) a été évaluée sur la série temporelle de photographies aériennes (entre 1934 et 2000) obtenues de l'Office fédéral de la topographie (Swisstopo), qui ont été scannées à une résolution de $50 \mathrm{~cm}$ par pixel, puis géoréférencées et orthorectifiées. Les photos anciennes étant en noir et blanc, toutes les images ont été analysées en échelle de gris. La méthode utilisée (Chételat et al, in print) a abouti à une classification structurale semi-automatique de la végétation à une résolution de $25 \mathrm{~m}$ en quatre catégories de taux de boisement (définies plus haut) selon Gallandat et al (1995) et Gillet \& Gallandat (1996). Ainsi, pour chacune des périodes 1934, 1954, 1968, 1985 et 2000, les proportions de superficie de chacune de ces catégories de taux de boisement ont pu être établies.

Le pâturage des Planets accuse au cours du temps une ségrégation marquée entre pâturages ouverts et forêts fermées (figure 5). Les pâturages faiblement boisés (2000) diminuent régulièrement de 1934 à 2000, un peu moins fortement entre 1985 et 2000. Cette catégorie a perdu plus de la moitié de sa surface initiale. Ce type de pâturage a été partiellement remplacé, soit par les pâturages non boisés (1000), spécialement entre 1934 et 1954, soit par les zones davantage boisées (3000 et 4000). Les pâturages non boisés (1000) ont augmenté régulièrement jusqu'en 1985 pour se stabiliser à environ 65\% de la 


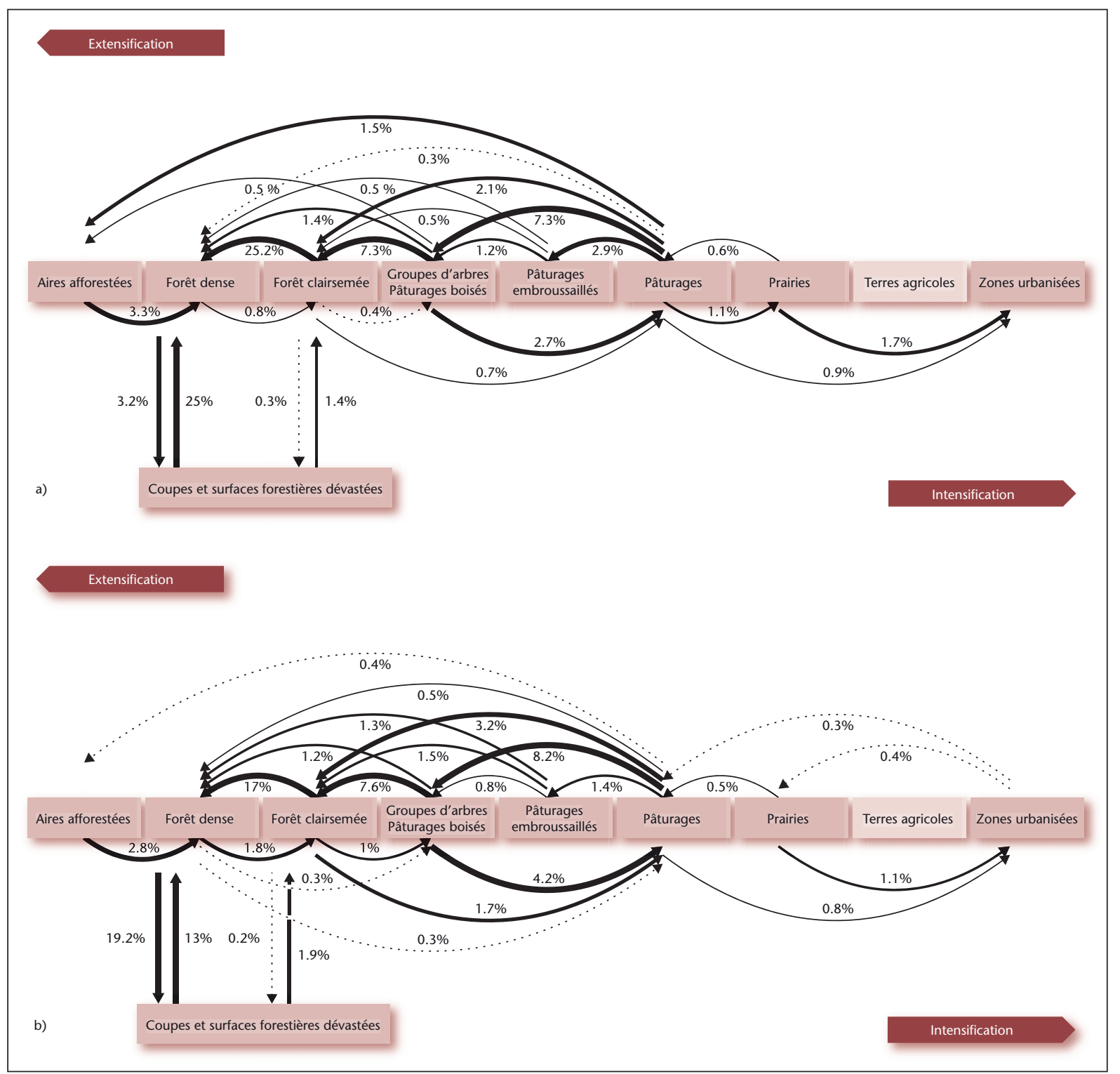

Fig. 4 Changement d'utilisation des sols dans les zones de montagne du Parc naturel régional Jura vaudois (à une altitude supérieure à 1000 m). A: période 1985 à 1997 , B: période 1997 à 2009. Les valeurs représentent les taux de changement en \% par rapport à l'ensemble des changements durant la période considérée (A: 1680 points; B: 1346 points). Les taux inférieurs à 0.2\% ne sont pas indiqués. La classe «Terres agricoles» est indiquée en beige, car il n’y a pas de point qui la concerne.

surface totale. Les pâturages densément boisées (3000) ont augmenté continuellement leur surface et en 2000 , leur proportion est devenue plus grande que celle des pâturages faiblement boisés (2000).

Le pâturage de la Rionde Dessus montre des proportions entre les quatre catégories de densité de boisement relativement similaires en 2000 par rapport à 1934 (figure 6). Avant 1968, une tendance à la réduction des pâturages densément boisés (3000) et des forêts pâturées (4000) est observée. En conséquence, entre 1934 et 1954, les pâturages faiblement boisés (2000) s'étendent, tandis que les pâturages non boisés (1000) restent constants. Entre 1954 et 1968, les pâturages non boisés (1000) ont augmenté considérablement au détriment de toutes les catégories de pâturages boisés, mais spécialement au détriment des pâturages faiblement boisés (2000). Après 1968, la tendance s'est inversée, avec une augmen- tation des pâturages densément boisés (3000) au détriment des pâturages non boisés (1000) et faiblement boisés (2000). Les forêts pâturées (4000) ont augmenté légèrement pendant cette période.

\section{Analyse prospective du paysage: modélisation et scénarios}

La question de l'évolution future du paysage sylvo-pastoral dans un contexte de changements climatiques a été abordée par une approche de modélisation et de simulation selon différents scénarios de changements climatiques et de gestion forestière. Le modèle dynamique spatialement explicite Wood PaM (Gillet 2008), qui intègre les interactions dans la mosaïque de végétation d'un pâturage boisé entre la gestion sylvo-,pastorale, l'activité du bétail, la dy- 


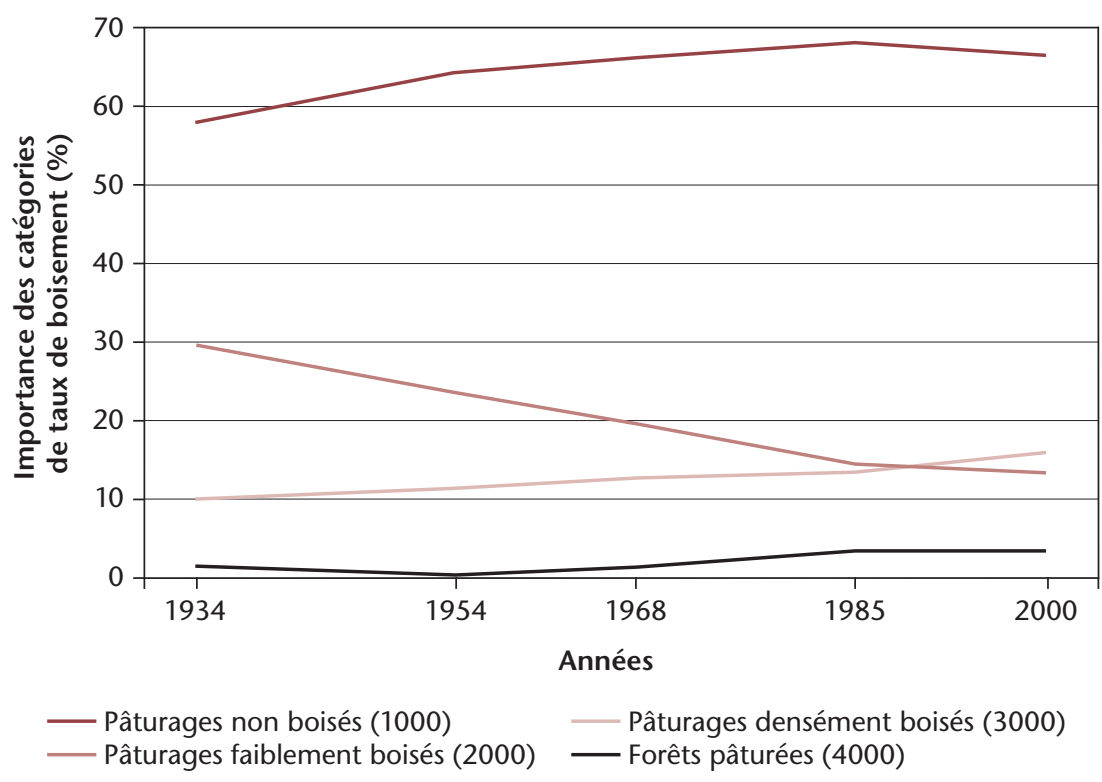

Fig. 5 Evolution des catégories de taux de boisement dans le pâturage Les Planets (Bullet) entre 1934 et 2000.

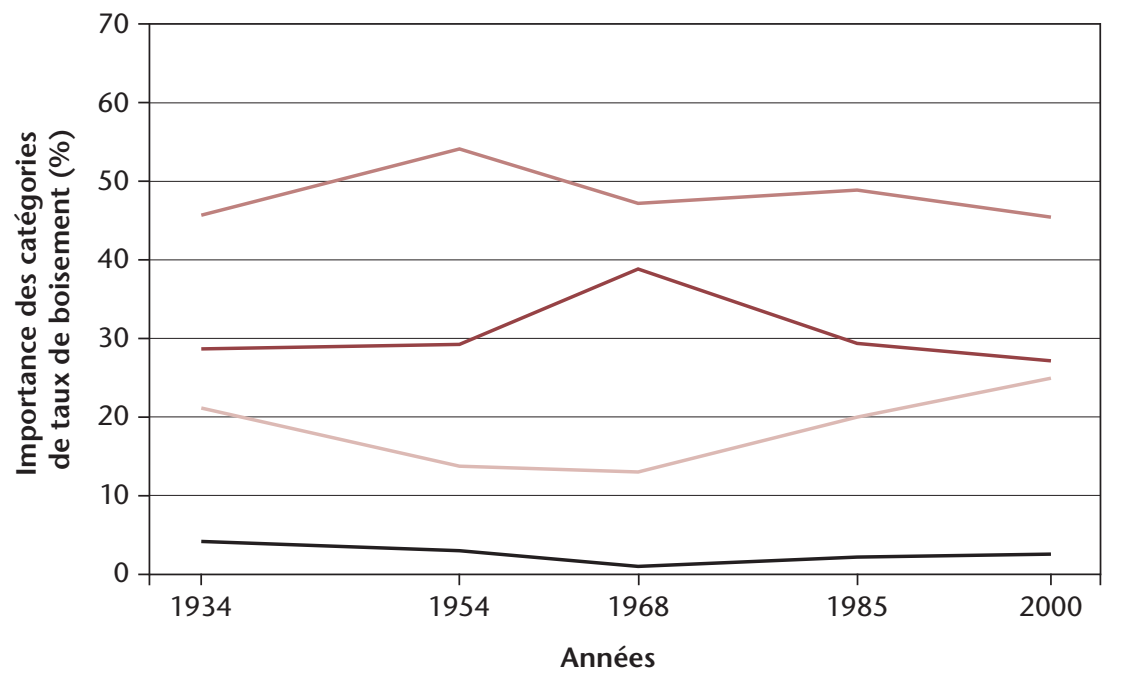

— Pâturages non boisés (1000)

— Pâturages densément boisés (3000) — Pâturages faiblement boisés (2000) —_ Forêts pâturées (4000)

Fig. 6 Evolution des catégories de taux de boisement dans le pâturage de La Rionde Dessus (Amburnex, Marchairuz) entre 1934 et 2000.

namique des herbages et la régénération des ligneux, a été affiné de manière à mieux représenter les processus démographiques des essences arborescentes (selon LandClim, Schumacher et al 2004, 2006, Schumacher \& Bugmann 2006), en tenant compte des fluctuations saisonnières de la température et

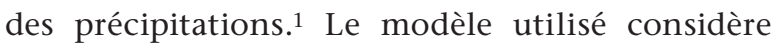
13 essences forestières issues du pool régional d'espèces (Picea abies, Abies alba, Pinus sylvestris, Fagus sylvatica, Quercus petraea, Q. robur, Q. pubescens, Carpinus betulus, Acer pseudoplatanus, A. campestre, Fraxinus excelsior, Tilia platyphyllos et Sorbus aucuparia).

L'évolution de différents pâturages a été simulée pour les conditions prédites par les scénarios climatiques B2 et A1FI de l'IPCC SRES (2000), soit une augmentation régionale des températures moyennes annuelles de $+4 \mathrm{~K}$, respectivement $+8 \mathrm{~K}$ entre 2001 et 2100 , la période de réchauffement, avec dans les deux cas une modification du régime des précipitations accentuant le déficit hydrique estival. Au-delà de cette période, les simulations ont été poursuivies jusqu'en 3000 en considérant des fluctuations aléatoires basées sur les variations de températures et précipitations mensuelles de la période 2091-2100. Après 2100, il est impossible d'établir un scénario réaliste et il s'agit uniquement d'analyser les tendances à long terme des changements de végétation induits par un siècle de changements climatiques (recherche d'un état d'équilibre). Pour cette modélisation, les conditions actuelles de gestion pastorale (charge en bétail, système de rotation et découpage des parcs) sont gardées telles qu'actuellement, et les prélèvements de bois ou les dégâts liés au tempêtes sont exclus (scénario NM; voir Peringer et al 2012 pour plus de détails sur le paramétrage du modèle). Additionnellement (Gillet \& Peringer 2012), deux scénarios de gestion forestière ont été testés pour chaque scénario climatique: la simulation d'une migration assistée par augmentation du taux fixe d'immigration de toutes les essences forestières pendant la période de réchauffement (scénario AM), et la simulation d'une exploitation forestière (scénario FM). Les résultats des simulations sont présentés ici sous forme de séries temporelles des quatre catégories de densité de boisement, du recouvrement cumulé des différentes essences arborescentes, d'un indice d'agrégation du paysage (He et al 2000) qui est une mesure inverse de l'hétérogénéité structurale du paysage, et de trois indices de diversité de Shannon: diversité des catégories de taux de boisement ou phytocénoses, des essences arborescentes (13 populations d'arbres) et des communautés herbacées ou synusies (moyenne de la diversité de quatre catégories de communautés herbacées dans chaque cellule du modèle spatialisé). Les résultats présentés concernent l'estivage du Pré aux Veaux, situé dans la combe des Amburnex, dans des conditions proches de celles de la Rionde (figure 2; voir Peringer et al, submitted, pour la comparaison de différents pâturages).

Les simulations montrent que l'impact des changements climatiques s'étale sur plusieurs siècles après la fin de la période de réchauffement et que l'évolution du paysage dépend fortement du scénario climatique dans le cas d'un scénario sans gestion forestière (figure 7). Jusqu'en 2050, seuls des changements faibles sont observés, avec un déclin des pâturages non boisés (1000) et le développement des forêts pâturées (4000). Durant cette période, l'épicéa reste largement dominant. Les deux scénarios pré-

\footnotetext{
1 PERINGER A ET AL (SUBMITTED) Past and future landscape dynamics in wooded pastures of the Jura Mountains under land-use and climate change. Ecol Soc.
} 


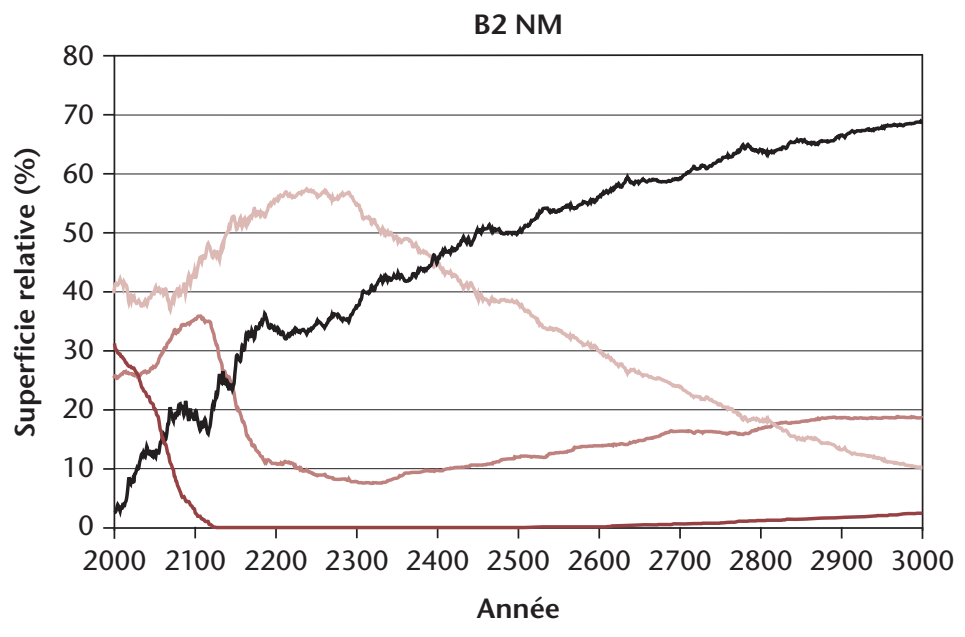

— Pâturages non boisés (1000) —Pâturages faiblement boisés (2000)

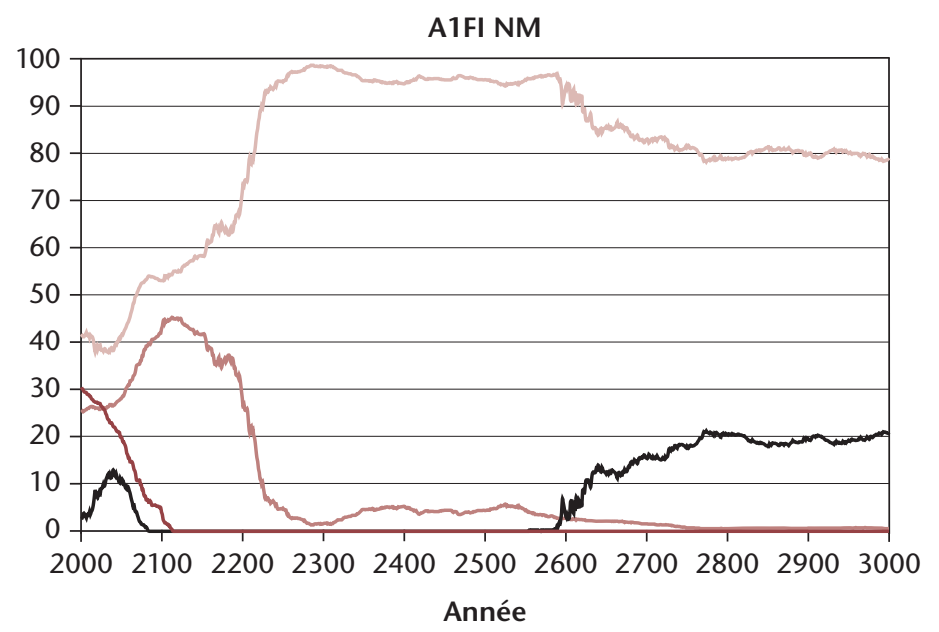

- Pâturages densément boisés (3000) — Forêts pâturées (4000)

Fig. 7 Simulation de la dynamique à long terme des catégories de taux de boisement dans le pâturage du Pré aux Veaux selon les scénarios climatiques B2 et A1FI sans gestion forestière (NM).
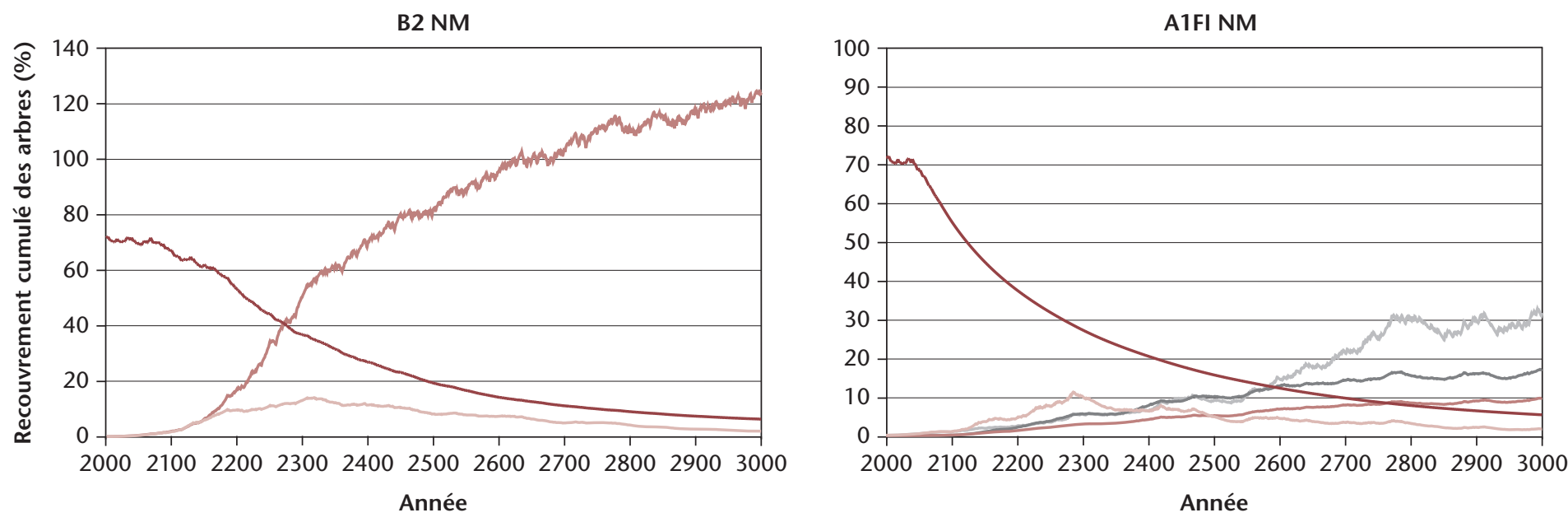

— Picea abies _ Fagus sylvatica _ Pinus sylvestris

Quercus pubescens _- Sorbus aucuparia

Fig. 8 Simulation de la dynamique à long terme des essences arborescentes dans le pâturage du Pré aux Veaux selon les scénarios climatiques B2 et A1FI sans gestion forestière (NM). Le recouvrement cumulé est calculé pour chaque essence à partir de sa densité dans les deux strates arborées (entre 1,5 et 8 m, et au-dessus de $8 \mathrm{~m}$ de hauteur) sur l'ensemble du paysage. Seules les espèces atteignant au moins 5\% de recouvrement cumulé sont représentées: Pa: Picea abies, Fs: Fagus sylvatica, Ps: Pinus sylvestris, Sa: Sorbus aucuparia, Qh: Quercus pubescens.

sentent par la suite une évolution divergente. Dans le scénario de réchauffement modéré B2, les forêts continuent à se développer lentement pour devenir dominantes à partir de 2400, au détriment des pâturages densément boisés (3000) qui régressent à partir de 2300. Dans le scénario extrême A1FI, les pâturages faiblement boisés (2000) et les forêts pâturées (4000) cèdent la place aux pâturages densément boisés (3000) qui dominent largement, avec cependant une réapparition des forêts à partir de 2600.

Les régressions temporaires du recouvrement des zones densément boisées entre 2050 et 2200 dans le scénario B2, et jusqu'en 2600 dans le scénario A1FI correspondent à un changement de composition de la strate arborescente (figure 8): dans les deux scénarios, l'épicéa, aujourd'hui largement dominant, décline continuellement. Dans le scénario B2, il est remplacé par le hêtre à partir de 2300. Dans le scénario A1FI, le remplacement est beaucoup plus lent et fait intervenir, outre le hêtre dans les secteurs peu pâturés (voir cartes dans Peringer et al 2012), le sorbier des oiseleurs, le pin sylvestre et le chêne pubescent dans les secteurs avec une pression plus élevée du bétail. Les autres essences ne jouent aucun rôle significatif dans le processus de succession initié par les changements climatiques.

L'indice d'agrégation tend à augmenter avec le temps, surtout pendant la phase initiale de réchauffement simulé (2001-2100), spécialement dans le scénario A1FI, traduisant la tendance générale à une simplification du paysage (figure 9). La migration assistée accélère ce processus, tandis que l'exploitation forestière permet de maintenir une certaine hétérogénéité spatiale à moyen terme, mais pas à long terme. A long terme, une exploitation forestière très 
B2

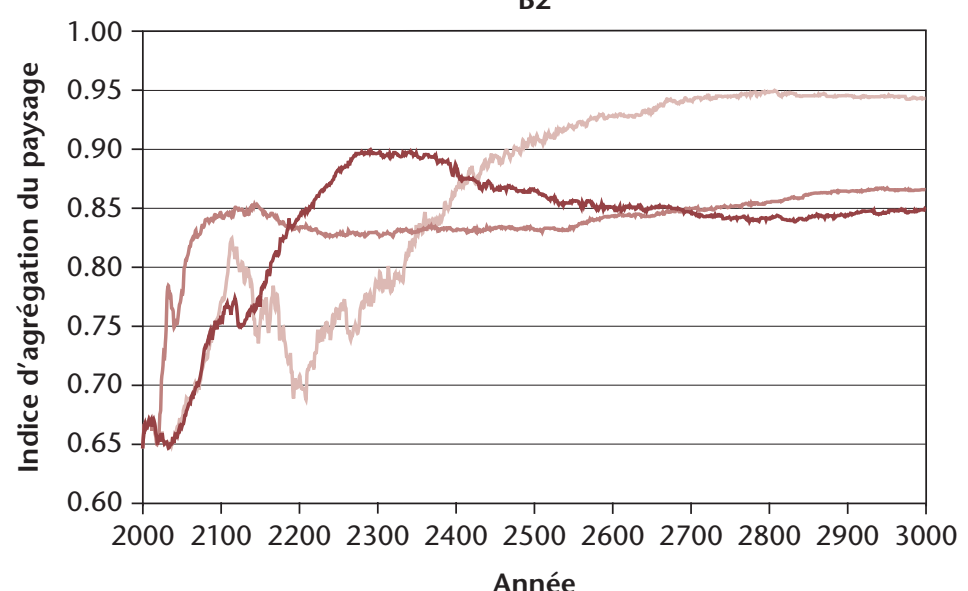

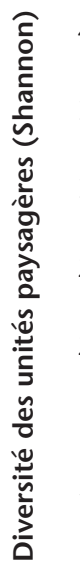

20002100220023002400250026002700280029003000

Année

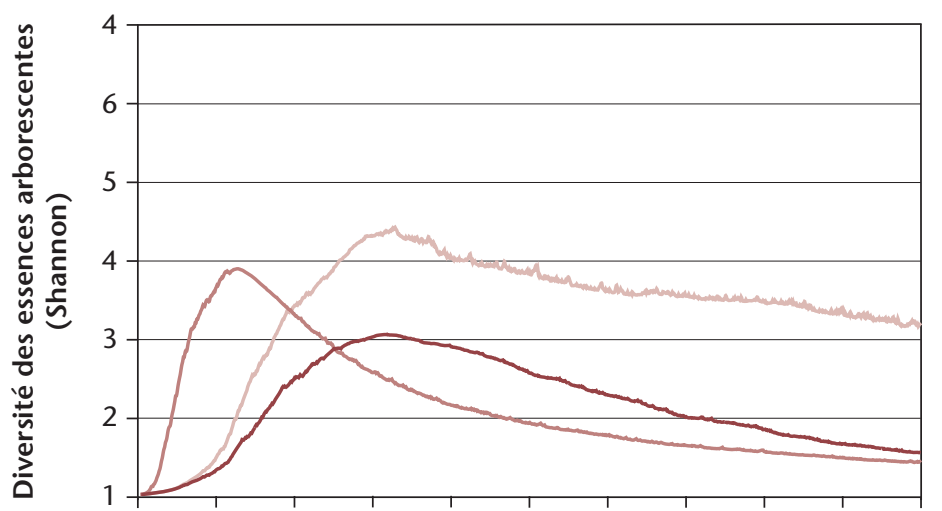

20002100220023002400250026002700280029003000

Année

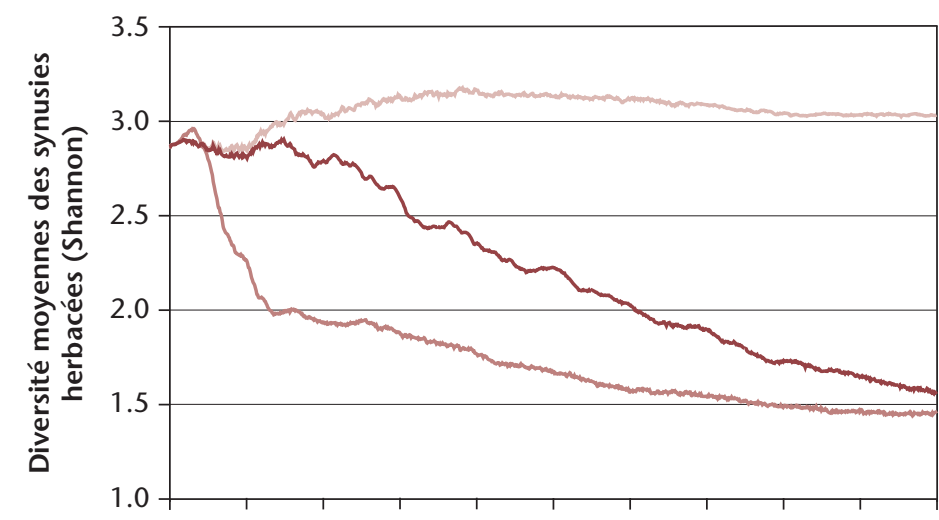

20002100220023002400250026002700280029003000

Année
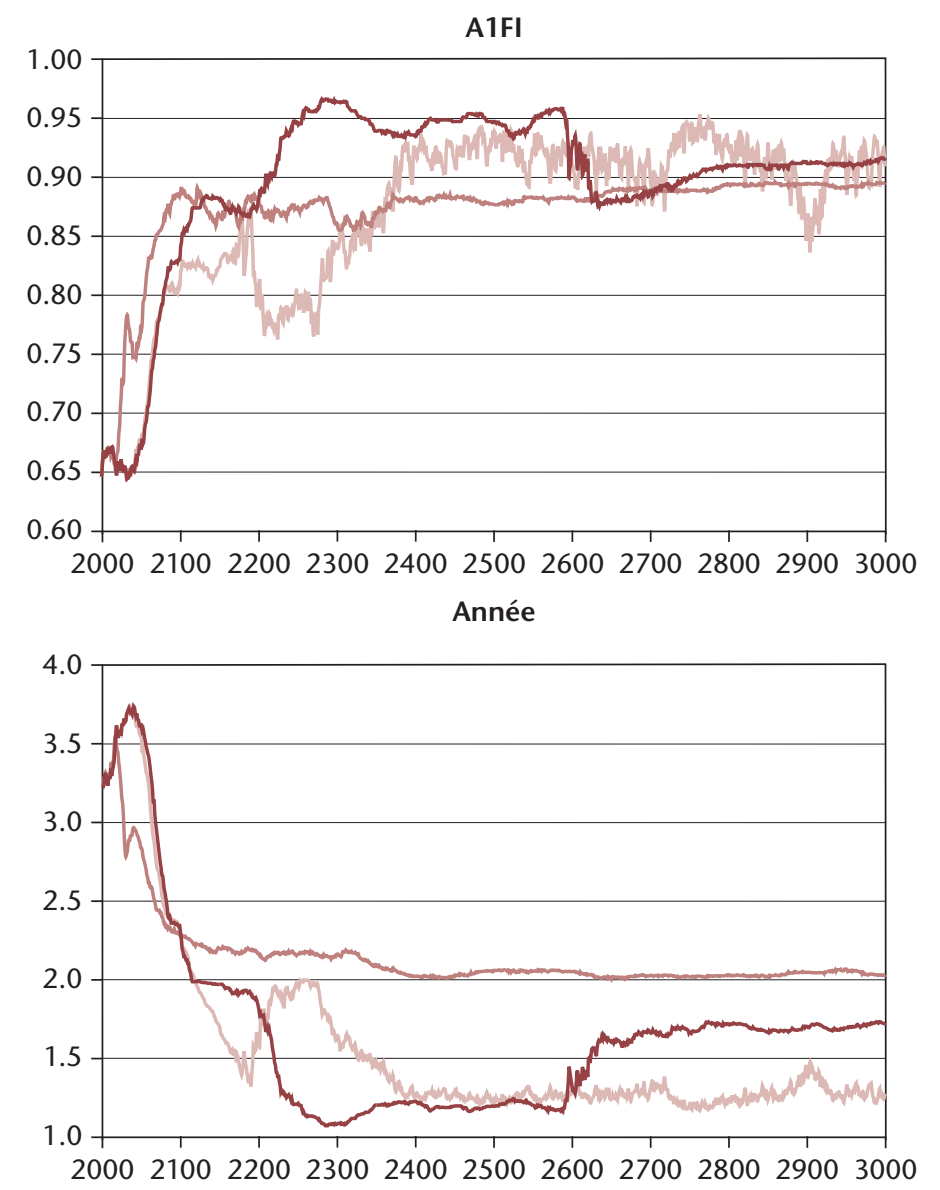

Année

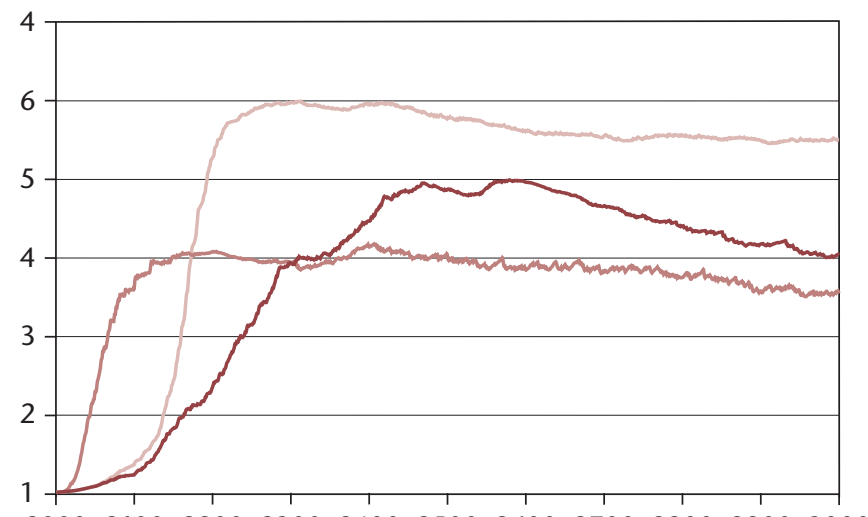

Année

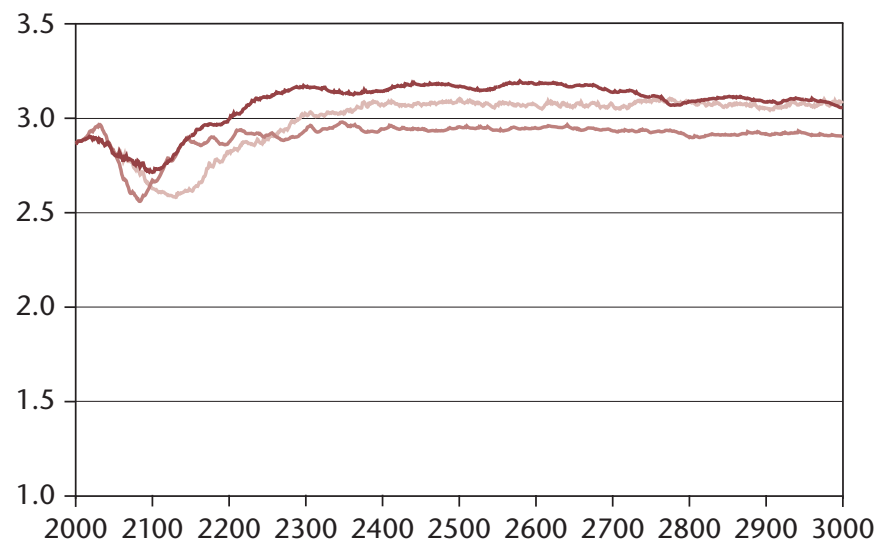

Année

$$
\text { - } \mathrm{NM}=\mathrm{AM}-\mathrm{FM}
$$

Fig. 9 Simulation de la dynamique à long terme des indices d'agrégation et de biodiversité du paysage dans le pâturage du Pré aux Veaux selon les scénarios climatiques B2 (à gauche) et A1FI (à droite) combinés à trois scénarios de gestion forestière (NM: aucune gestion; AM: migration assistée; FM: exploitation des arbres). 
dirigée pourrait introduire des cycles de régénération et une mosaique spatio-temporelle dans les forêts de pin sylvestre (scénario A1FI), comme c'était le cas avec la forêt d'épicéa.

La diversité des unités paysagères augmente pendant la période de réchauffement simulé (20012100), puis diminue ensuite quelle que soit l'intensité de celui-ci (figure 9). Le déclin est plus rapide et drastique pour le scénario A1FI. La migration assistée supprime le pic de diversité au XXI' siècle dans les deux scénarios climatiques, mais limite son déclin à long terme dans le scénario A1FI. A long terme, dans ce scénario, l'exploitation forestière a plutôt un effet négatif sur la diversité paysagère.

La diversité des essences dans la strate arborescente (figure 9), très faible au départ (hyper-dominance de l'épicéa), augmente dans tous les scénarios, plus ou moins rapidement selon la gestion forestière, et reste très élevée à long terme pour le scénario A1FI. L'exploitation forestière a un effet positif sur la diversification des essences parce qu'elle diminue la dominance du hêtre ou du pin, selon le scénario climatique considéré.

La diversité moyenne des communautés herbacées (figure 9), supposée favoriser la biodiversité végétale, tend à diminuer pendant la phase de réchauffement (2001-2100) pour tous les scénarios. En absence de gestion forestière, elle continue à décroître dans le scénario B2, tandis qu'elle se stabilise à une valeur élevée dans le scénario A1FI. La migration assistée des essences arborescentes a un effet négatif sur la diversité des synusies herbacées dans le scénario B2, tandis que l'exploitation forestière permet d'empêcher sa décroissance.

\section{Discussion et conclusion}

L'analyse historique a montré une certaine résilience de la structure des paysages sylvo-pastoraux dans la région de montagne du Jura vaudois par rapport aux zones de plus basse altitude, malgré une tendance à la ségrégation entre forêts fermées et pâturages. La gestion pastorale et sylvicole, ainsi que les événements climatiques extrêmes (tempêtes) ont provoqué au cours du XX $\mathrm{X}^{\mathrm{e}}$ siècle des fluctuations de la structure paysagère sans que celle-ci n'ait subi des ruptures sensibles. Chételat et al (in print) ont montré à l'aide de représentations cartographiques que ces changements ont été particulièrement importants spatialement durant la période 1934-1954 (exploitation plus intense après la guerre, dégâts dus aux tempêtes et à la sécheresse). La représentation non spatiale de l'évolution de la couverture du boisé dans les deux sites (figures 5 et 6) montre que durant la période 1954-1968, notamment suite à la politique agricole protectioniste et les subsides à la production, de même qu'en raison de l'amélioration des techniques agricoles, l'augmentation des pâturages ouverts a été significative. Ce phénomène a été particulièrement visible sur le site de La Rionde Dessus, mais uniquement temporairement, pour laisser ensuite une densification du boisé en lien avec les mesures incitatives à la limitation de la production laitière et le contingentement laitier dans les années 1980. Sur le site Les Planets, cette tendance à l'ouverture à été plus progressive et durable en raison de la possibilité d'une meilleure rationalisation de l'exploitation près du village, conduisant à une ségrégation entre zones ouvertes et zones fermées.

Les simulations de l'évolution future de ces paysages suggèrent qu'il faut s'attendre à des changements radicaux dans la composition et la structure de la végétation, provoqués par les changements climatiques en cours, même si ces changements resteront peu visibles à court terme. Sur le long terme, les zones à forte densité de boisé auront tendance à s'étendre, et l'épicéa cédera la place au hêtre ou au pin, selon l'intensité du réchauffement. L'exploitation forestière sera nécessaire pour aller à l'encontre de la simplification des paysages et pour maintenir une certaine diversité paysagère. Cependant, l'adaptation à ces nouvelles conditions environnementales pour assurer la durabilité des services des écosystèmes nécessitera des mesures de gestion qui dépendront elles-mêmes de l'intensité des changements climatiques. La migration assistée et l'exploitation forestière pourront avoir des effets positifs ou négatifs selon le critère considéré (structure du paysage, diversité des essences ou des espèces herbacées) et selon l'intensité du réchauffement. Seule une gestion adaptative active, basée sur l'expérimentation et l'innovation, mais qui se fait aussi avec la connaissance de l'histoire d'exploitation et les expériences passées, et associant scientifiques et gestionnaires, permettra de limiter l'ampleur des effets des changements climatiques. La politique agricole et les réglementations devront aussi s'accommoder de la nécessité d'une gestion différenciée dans l'espace, de manière à pouvoir tenir compte des processus locaux, et permettre des mesures de gestion ciblées. A cet égard, la simulation des effets combinés des changements climatiques et de la réforme agraire PA1417 montre que la conversion des paiements directs à la surface gérée plutôt qu'au nombre de têtes de bétail favorise l'extensification des pâturages boisés, mais que, en même temps, les conditions climatiques vont renforcer la compétitivité des arbres, ce qui, à plus long terme, favorisera la ségrégation entre pâturages ouverts et forêt fermée (Buttler et al 2012). Mais le pouvoir régulateur du microclimat dans les pâturages plus ou moins boisés et, en conséquence, le maintien d'une bonne production fourragère malgré le réchauffement climatique et la sécheresse sont également mis en évidence par les simulations des modèles dynamiques (Gavazov et al, in print), ce qui 
pourrait rendre les pâturages boisés de montagne encore plus attractifs et augmenter la pression de pâture.

Afin de conserver à l'avenir les diverses fonctions des pâturages boisés et la diversité du paysage jurassien, malgré les changements climatiques, des mesures complémentaires devront être envisagées dans le cadre de la nouvelle PA14-17 dans le but de permettre une pression de pâture adaptée à la station et de réduire de manière ciblée l'avancement de la forêt. L'adaptation du zonage en surface agricole utile (SAU) ou zone d'estivage pourrait s'avérer judicieuse pour une gestion adaptative de ces écosystèmes.

Soumis: 11 avril 2012, accepté (avec comité de lecture): 3 octobre 2012

\section{Références}

BARBEZAT V, BOQUET JF, EDITORS (2008) Gestion intégrée des paysages sylvo-pastoraux de l'Arc jurassien - Manuel (Handbook). La Chaux-de-Fonds: Conférence TransJurassienne. 160 p.

BUTTLER A, KOHLER F, GILLET F (2009) The Swiss mountain wooded pastures: patterns and processes. In: Rigueiro-Rodrigues A, McAdam J, Mosquera-Losada MR, editors. Agroforestry in Europe: current status and future prospects. Dordrecht: Springer. pp. 377-396.

BUTTLER A ET AL (2012) Conservation des pâturages boisés du Jura: défis climatiques et agro-politiques. Recherche Agronomique Suisse 3: 346-353.

CHÉTELAT J ET AL (IN PRINT) A contextual analysis of observed land-use and vegetation changes applied to two wooded pastures in the Swiss Jura Mountains. Ecol Soc.

DUFOUR A, GADALLAH Z, WAGNER H, GUISAN A, BUTTLER A (2006) Plant species richness and environmental heterogeneity in a mountain landscape: effects of variability and spatial configuration. Ecography 29: 573-584.

ETIENNE M (1996) Western European silvopastoral systems. Paris: INRA. 276 p.

GALLANDAT JD, GILLET F, HAVLICEK E, PERRENOUD A (1995) Typologie et systémique phyto-écologiques des pâturages boisés du Jura suisse. Laboratoire d'écologie végétale, Neuchâtel: Université de Neuchâtel. 466 p.

GAVAZOV K, PERINGER A, BUTTLER A, GILLET F, SPIEGELBERGER T (IN PRINT) Dynamics of forage production in pasture-woodlands of the Swiss Jura Mountains under projected climate change scenarios. Ecol Soc.

GILLET F, GALLANDAT JD (1996) Wooded pastures of the Jura mountains. In: Etienne M, editor. Western european silvopastoral systems. Paris: INRA. pp. 37-53.

GILLET F, MURISIER B, BUTTLER A, GALLANDAT JD, GOBAT JM (1999) Influence of tree cover on the diversity of herbaceous communities in subalpine wooded pastures. Appl Veg Sci 2: 47-54.

GILLET F (2008) Modelling vegetation dynamics in heterogeneous pasture-woodland landscapes. Ecol Model 217: 1-18.

GILLET F, KOHLER F, VANDENBERGHE C, BUTTLER A (2010) Effect of dung deposition on small-scale patch structure and seasonal vegetation dynamics in mountain pastures. Agric Ecosyst Environ 135: 34-41.

GILLET F, PERINGER A (2012) Dynamic modelling of silvopastoral landscape structure: Past reconstruction and scenarios for future climate and land use. www.isa.utl.pt/def/fp0603forestmodels/FM/08-GILLET.pdf (4.11.2012).
GMÜR P, WETTSTEIN JB (1986) L'aménagement des pâturages communaux dans les Franches-Montagnes (Canton du Jura). Rev Suisse Agric 18: 87-92.

GMÜR P, THOMET P, WETTSTEIN JB (1989) Etablissement d'un plan de gestion d'un pâturage du Jura vaudois: planification de la cohabitation d'espaces naturels et de surfaces productives. Zollikofen: Technicum agricole suisse.

GOBAT JM, DUCKERT O, GALLANDAT JD (1989) Quelques relations microtopographie-sols-végétation dans les pelouses pseudo-alpines du Jura suisse: exemples d'un système naturel et d'un système anthropisé. Bull soc neuchâtel sci nat 112: 5-17.

HAVLICEK E, GOBAT JM, GILLET F (1998) Reflections on relationships between vegetation and soil: three examples on allochtonous material in the Jura Mountains. Ecologie 4: 535-546.

HE HS, DEZONIA BE, MLADENOFF DJ (2000) An aggregation in$\operatorname{dex}(\mathrm{Al})$ to quantify spatial patterns of landscapes. Landsc Ecol 15: 591-601.

IPCC WG III (2000) Emissions scenarios - summary for policymakers. Geneva: Intergovernmental Panel on Climate Change, Special Report. 27 p.

KOHLER F, GILLET F, PROGIN MA, GOBAT JM, BUTTLER A (2004A) Seasonal dynamics of plant species at fine scale in wooded pastures. Community Ecol 5: 7-17.

KOHLER F, GILLET F, GOBAT JM, BUTTLER A (2004B) Seasonal vegetation changes in mountain pastures due to simulated effects of cattle grazing. J Veg Sci 15: 143-150.

KOHLER F, HAMELIN J, GILLET F, GOBAT JM, BUTTLER A (2005) Soil microbial community changes in wooded mountain pastures due to simulated effects of cattle grazing. Plant Soil 278: 327-340.

KOHLER F ET AL (2006A) Spatial and seasonal patterns of cattle habitat use in a mountain wooded pasture. Landsc Ecol 21: 281-295.

KOHLER F, GILLET F, GOBAT JM, BUTTLER A (2006B) Effect of cattle activities on gap colonisation in mountain pastures. Folia Geobot 41: 289-304.

MIEVILLE-OTT V, BARBEZAT V (2005) Perception du pâturage boisé: résultats d'un sondage effectué au Communal de la Sagne NE. Schweiz Z Forstwes 156: 1-12. doi: 10.3188/ szf.2005.0001

OFS (2001) The changing face of land-use: Land use statistics of Switzerland. Technical report. Neuchâtel: Swiss Federal Statistical Office. 32 p.

PERRENOUD A, KÄNZIG-SCHOCH U, SCHNEIDER O, WETTSTEIN JB (2003) Exploitation durable des pâturages boisés. Un exemple appliqué du Jura suisse. Bern: Haupt. 235 p.

SCHUMACHER S, BUGMANN H, MLADENOFF DJ (2004) Improving the formulation of tree growth and succession in a spatially explicit landscape model. Ecol Model 180: 175-194.

SCHUMACHER S, BUGMANN H (2006) The relative importance of climatic effects, wildfires and management for future forest landscape dynamics in the Swiss Alps. Glob Chang Biol 12: 1435-1450.

SCHUMACHER S, REINEKING B, SIBOLD J, BUGMANN H (2006) Modeling the impact of climate and vegetation on fire regimes in mountain landscapes. Landsc Ecol 21: 539-554.

SMIT C, BÉGUIN D, BUTTLER A, MÜLLER-SCHÄRER H (2005) Safe sites for tree regeneration: a case of associational resistance? J Veg Sci 16: 209-214.

VANDENBERGHE C, FRELECHOUX F, GADALLAH F, BUTTLER A (2006) Competitive effects of herbaceous vegetation on tree seedling emergence, growth and survival: does gap size matter? J Veg Sci 17: 481-488.

VANDENBERGHE C, FRELÉCHOUX F, BUTTLER A (2007A) The influence of shade and competition from herbaceous vegetation on simulated browsing tolerance of coniferous and deciduous saplings. Oikos 117: 415-423. 
VANDENBERGHE C, FRELECHOUX F, MORAVIE MA, GADALLAH F, BUTTLER A (2007B) Short-term effects of cattle browsing on tree sapling growth in mountain wooded pastures. Plant Ecol 188: 253-264.

VANDENBERGHE C, SMIT C, POHL M, BUTTLER A, FRELECHOUX $F$ (2009) Does the strength of facilitation by nurse shrubs depend on grazing resistance of tree saplings? Basic Appl Ecol 10: $427-436$

\section{Evolution récente et future des paysages sylvo-pastoraux du Jura vaudois}

L'utilisation des paysages sylvo-pastoraux est particulièrement délicate, car leur existence dépend du subtil équilibre de leur gestion qui vise à fournir différents biens et services écologiques, allant de la production d'herbe et de bois à la promotion de la biodiversité, ainsi qu'à la mise à disposition d'aires de détente et de paysages attractifs. Dans cet article, nous présentons une analyse rétrospective, basée sur les données statistiques du territoire, des changements d'utilisation des sols du Parc naturel régional Jura vaudois entre 1985 et 2009, et une analyse rétrospective de la couverture du boisé dans deux pâturages du Jura vaudois par analyse de cinq séries de photographies aériennes anciennes entre 1934 et 2000. Une modélisation prospective sur le long terme (2001 à 3000) est ensuite présentée pour un pâturage de manière à évaluer les changements de paysage selon deux scénarios climatiques de réchauffement et trois variantes de gestion forestière. Les résultats de l'analyse rétrospective montrent une certaine résilience de la structure des paysages sylvo-pastoraux dans la région de montagne du Jura vaudois par rapport aux zones de plus basse altitude, malgré une tendance à la ségrégation entre forêts fermées et pâturages. Les politiques agricoles ont marqué la dynamique des taux de boisement, mais de manière différenciée selon la situation locale, par exemple celle de la proximité d'un village qui permet une meilleure rationalisation de l'exploitation, ou celle de l'éloignement qui favorise l'abandon. Les simulations de l'évolution future de ces paysages suggèrent qu'il faut s'attendre à des changements radicaux dans la composition et la structure de la végétation, provoqués par les changements climatiques. Sur le long terme, les zones à forte densité de boisé auront tendance à s'étendre, et l'épicéa cédera la place au hêtre et/ou au pin sylvestre, selon l'intensité du réchauffement. Pour assurer la durabilité des services des écosystèmes, l'adaptation à ces nouvelles conditions environnementales nécessitera des mesures de gestion qui dépendront ellesmêmes de l'intensité des changements climatiques. Seule une gestion adaptative active, basée sur l'expérimentation et l'innovation, et associant scientifiques et gestionnaires, permettra de limiter l'ampleur des effets des changements climatiques.
VITTOZ P (1998) Flore et végétation du Parc jurassien vaudois: typologie, écologie et dynamique des milieux. Lausanne: Univ Lausanne, PhD Thesis. $446 \mathrm{p}$.

\section{Bisherige und künftige Entwicklung der Wytweidenlandschaft im Waadtländer Jura}

Die Nutzung der Wytweiden ist anspruchsvoll, da ihre Existenz vom subtilen Gleichgewicht in ihrer Bewirtschaftung abhängt. Die Bewirtschaftung ist dabei auf die Bereitstellung verschiedener Ökosystemleistungen ausgerichtet, so die Produktion von Gras und Holz, die Förderung der Biodiversität sowie die Bereitstellung von Erholungsgebieten und attraktiven Landschaften. Im vorliegenden Artikel präsentieren wir die Ergebnisse von Untersuchungen, die anhand der schweizerischen Arealstatistik Landnutzungsänderungen im regionalen Naturpark «Jura vaudois» zwischen 1985 und 2009 sowie Veränderungen des Bestockungsgrads in zwei Wytweiden des Waadtländer Juras mithilfe von fünf Luftbildserien zwischen 1934 und 2000 analysieren. Weiter modellieren wir die langfristigen Landschaftsveränderungen (2001 bis 3000) in einer Wytweide aufgrund von zwei Klimaszenarien und drei forstlichen Bewirtschaftungsvarianten. Die retrospektiven Analysen zeigen eine zunehmende Trennung zwischen geschlossenem Wald und Weiden, wobei die Veränderungen in den höheren Lagen des Waadtländer Juras weniger stark sind als in den tieferen Lagen. Dabei bestimmt die Agrarpolitik die Veränderungen im Bestockungsgrad und zwar differenziert nach den örtlichen Gegebenheiten. So werden beispielsweise Weiden in Dorfnähe rationeller bewirtschaftet, entfernte Weiden hingegen aufgegeben. Die Modellierung der zukünftigen Landschaftsentwicklung deutet darauf hin, dass sich im Zuge des Klimawandels die Zusammensetzung und die Struktur der Vegetation radikal verändern werden. Auf lange Sicht dürften sich die dicht bestockten Bereiche ausdehnen, und je nach Klimaszenario dürfte die Buche und/oder die Waldföhre die Fichte verdrängen. Um die Wytweiden an die neuen Umweltbedingungen anzupassen, sind Massnahmen nötig, welche ihrerseits abhängig sind von der Intensität des Klimawandels. Nur ein adaptives und aktives Management, welches auf Experimenten und Innovationen basiert sowie Wissenschaft und Praxis verbindet, wird es erlauben, die Effekte des Klimawandels abzumildern. 